\title{
Os efeitos de diferentes pesos de mochila no alinhamento postural de crianças em idade escolar.
}

\author{
The effects of different backpacks weights on postural alignment of \\ children of school age
}

\author{
L.G. Ries, M. Martinello, M. Medeiros, M. Cardoso, G.M. Santos
}

ARTIGO ORIGINAL | ORIGINAL ARTICLE

\begin{abstract}
RESUMO
O objetivo deste estudo foi analisar o efeito imediato de diferentes pesos de mochila na postura corporal e avaliar a confiabilidade dessas medidas. Foram avaliados 50 escolares com idades entre oito e 14 anos. Os escolares foram fotografados no plano coronal anterior e sagital, com diferentes cargas relativas ao peso: sem mochila, com o peso habitualmente utilizado, com $10 \%$ do peso corporal (PC), $15 \%$ do PC e $20 \%$ do PC. Para comparar os ângulos de assimetria da cabeça, de ombros, pélvica, do trocanter, dos joelhos, maleolar, corporal, sagital da cabeça, sagital dos ombros e sagital corporal foi utilizada a Análise de Variância para Medidas Repetidas e o Teste de Friedman. A confiabilidade foi analisada por meio do Coeficiente de Correlação Intraclasse (ICC). Foram observadas diferenças estatisticamente significativas no ângulo sagital cervical $(p<.00)$, no ângulo sagital corporal $(p<.00)$ e no ângulo sagital dos ombros $(p<.01)$ com o aumento da carga. Também obteve-se alta confiabilidade inter-avaliador (ICC entre .83 a .99) para todos os ângulos avaliados nas diferentes posturas $(p<.05)$. Este estudo não sustenta a recomendação de que o peso da mochila não deve exceder $10 \%$ do PC, já que cargas inferiores também ocasionaram anteriorização da cabeça e do corpo.

Palavras-chave: postura, peso corporal, estudantes
\end{abstract}

ABSTRACT

The aim of this study was to analyze the acute effect of different weights of backpack on body posture and assess the reliability of these measures. Were evaluated 50 students aged between eight and 14 years old. The students were photographed in the coronal and sagittal planes with different backpack loads: no backpack, the weight commonly used, with $10 \%, 15 \%$ and $20 \%$ of body weight. To compare the angles of asymmetry of the head, shoulders, pelvis, trochanter, knee, malleolus, body, sagittal head, shoulder sagittal and sagittal body was used ANOVA for repeated measures and the Friedman test. Reliability was analyzed using the intraclass correlation coefficient (ICC). Statistically significant difference were observed in the sagittal angle of the head $(p<.00)$, in the sagittal body angle $(p<.00)$ and the sagittal angle of the shoulders $(p<.01)$ with increasing load. The data shows high inter-rater reliability (ICC $>.83$ ) for all angles measured in different postures $(p<.05)$. This study does not support the recommendation that the weight of the backpack should not exceed $10 \%$ of body weight since smaller loads also caused anterior displacement of the head and body.

Keywords: posture, body weight, students

Submetido: 04.02.2012 | Aceite: 02.12.2012

Lílian Gerdi Kittel Ries, Micheli Martinello, Melina Medeiros, Mabel Cardoso, Gilmar Moraes Santos. Universidade do Desenvolvimento do Estado de Santa Catarina - UDESC - Santa Catarina, SC - Brasil.

Endereço para correspondência: Lílian Gerdi Kittel Ries, Rua Pascoal Simone, 358, Bairro Coqueiros, Florianópolis - SC, CEP 88080-350, Brasil.

E-mail:d2lgkr@udesc.br; liliangkr@yahoo.com.br 
A utilização de mochilas escolares pela população pediátrica vem gerando discussões sobre o impacto diário na postura corporal devido ao peso transportado. O peso transportado pelos escolares em suas mochilas predispõe a um desequilíbrio músculo esquelético causado pelo deslocamento posterior do centro de massa (Al-Khabbaz, Shimada, \& Hasegawa, 2008). No intuito de manter o corpo em equilíbrio surgem compensações e assimetrias posturais (Al-Khabbaz et al., 2008), bem como mudanças na base de apoio e na pressão arterial (Hong \& Brueggmann, 2000).

Diversos pesquisadores têm se preocupado em determinar um percentual em relação ao próprio peso corporal da criança que minimize a instalação de alterações posturais. A literatura, como um todo, recomenda o percentual de $10 \%$ e de $15 \%$ (Whittifielf, Legg, \& Hedderley, 2005). Contudo, alguns estudos questionam a utilização desses percentuais e relacionam-nos com um trabalho excessivo de determinados grupos musculares e a alterações significativas nos ângulos articulares durante as atividades diárias (Hong \& Brueggmann, 2000; Moore, White, \& Moore, 2007; Seven, Akalan, \& Yucesoy, 2008). Transportar mochilas com peso excessivo e de forma habitual apresenta um impacto cumulativo na postura dessas crianças.

A relação entre o transporte de carga nas mochilas e a alta incidência de desvios e de compensações posturais observadas em crianças desperta a necessidade de quantificar os prejuízos advindos desta situação e trabalhar junto aos escolares principalmente no âmbito preventivo (Goodgold et al., 2002; Hong \& Brueggmann, 2000; Seven et al., 2008). Nas crianças, o sistema músculo esquelético é complacente e o diagnóstico, assim como a prevenção precoce, além de auxiliar na correção das alterações já instaladas evitam o aparecimento de alterações secundárias a estes desvios posturais ou ao agravamento dos mesmos (Santos et al., 2009).

Entende-se que são inúmeros os danos ocasionados pelo transporte excessivo de material escolar. Portanto, a preocupação com a saúde escolar é determinante para o crescente desenvolvimento de pesquisas relacionadas com o tema. Embora haja recomendações sobre os limites de peso das mochilas escolares, a literatura é controversa sobre os seus efeitos no equilíbrio músculo esquelético da população pediátrica. Diante do exposto, este estudo teve como objetivos analisar o efeito imediato de diferentes pesos de mochila na postura corporal, assim como avaliar a confiabilidade dessas medidas.

\section{MÉTODO}

\section{Amostra}

A população deste estudo foi composta por escolares na faixa etária de oito a 14 anos, matriculados no ensino fundamental da rede estadual de ensino da cidade de Florianópolis. A amostra foi constituída por 50 escolares, de ambos os sexos (20 do sexo feminino e 30 do sexo masculino). As características antropométricas dos escolares são apresentadas na Tabela 1.

Tabela 1

Caracterização da amostra $(n=50)$

\begin{tabular}{ccc}
\hline & Média & DP \\
\hline Idade (anos) & 10.32 & 1.73 \\
Peso (kg) & 38.04 & 9.65 \\
Altura (m) & 1.42 & 0.14 \\
\hline
\end{tabular}

Para inclusão dos escolares utilizaram-se os seguintes critérios: IMC normal, sem patologias associadas ou má formação congênita de membros inferiores, não portadores de necessidades especiais (neurológica e/ou física) e não participantes de qualquer tratamento ortopédico e/ou fisioterápico.

Os responsáveis foram informados sobre as atividades desenvolvidas, assim como seus objetivos. Para a participação dos escolares, um Termo de Consentimento Livre e Esclarecido (TCLE) foi assinado pelos pais ou responsáveis. 


\section{Instrumentos}

Os instrumentos utilizados no presente estudo foram a anamnese e a fotogrametria. Por meio da anamnese realizou-se a caracterização da amostra, constando de dados antropométricos, fatores de exclusão e verificação do peso de mochila habitualmente utilizado pela criança.

A fotogrametria é considerada uma avaliação postural quantitativa, utilizada para medidas lineares e angulares (Iunes et al., 2005). Para a fotogrametria foi utilizada uma câmara digital Sony® 7.2 Megapixels, posicionada em solo nivelado, sobre um tripé a uma altura de 0.85 $\mathrm{cm}$ e a uma distância de $2.4 \mathrm{~m}$ do indivíduo avaliado. A análise postural foi realizada por meio do software SAPO, validado por Ferreira, Duarte, Maldonado, Burke, e Marques (2010). É um programa amplamente utilizado pelos profissionais de saúde, constituindo-se como um passo inicial e de acompanhamento para a avaliação e tratamento clínico.

\section{Procedimentos}

Em seguida à anamnese, realizou-se a avaliação postural, na qual os participantes vestiam trajes adequados e adotavam a posição ortostática com os braços ao longo do corpo e pés na postura preferencial. A posição dos pés adotada pela criança foi registrada, pois o posicionamento inicial e a postura preferencial deviam ser mantidos durante toda a sequência das fotos.

$\mathrm{Na}$ avaliação postural sinalizaram-se as referências anatômicas por meio de marcadores esféricos com $1 \mathrm{~cm}$ de diâmetro, de coloração branca, fixados por fita de dupla face à superfície corporal. Utilizou-se um fio de prumo como eixo vertical de referência. A mochila de duas alças foi posicionada nos dois ombros, da forma normalmente utilizada pela criança, com diferentes cargas relativas ao peso: sem mochila, com o peso habitualmente utilizado, com $10 \%$ do PC, $15 \%$ do PC e $20 \%$ do PC. A ordem dos pesos foi determinada alea- toriamente. As imagens, no plano coronal e lateral direito, foram registradas por um único avaliador. Um segundo avaliador foi responsável pela marcação dos pontos nas seguintes referências anatômicas: cervical (C7), glabela, tragos, acrômios, espinhas ilíacas ântero-superiores, trocânter maior do fêmur, linhas articulares do joelho e maléolos laterais.

A digitalização das imagens fotográficas e o cálculo dos ângulos foram realizados por três avaliadores diferentes. Todos os avaliadores foram previamente treinados. Os seguintes ângulos foram analisados no plano coronal anterior, sendo definidos como "assimetrias": assimetria da cabeça, assimetria de ombros, assimetria pélvica, assimetria do trocanter, assimetria dos joelhos, assimetria maleolar (todos os ângulos são formados pela linha que passa pelos marcadores do lado esquerdo e direito de cada referência anatômica e linha perpendicular ao fio de prumo) e assimetria corporal (ângulo livre formado pela linha que passa pelo marcador da glabela e ponto médio entre os maléolos com linha paralela ao fio de prumo que passa no ponto médio entre os maléolos). No plano sagital: ângulo sagital cervical (ângulo livre formado pela linha que passa no marcador do trago ao marcador da C7 e linha perpendicular ao fio de prumo que passa no marcador da C7), ângulo sagital dos ombros (ângulo livre formado pela linha que passa no marcador da C7 ao marcador do acrômio e linha perpendicular ao fio de prumo que passa no marcador do acrômio) e ângulo sagital corporal (ângulo livre formado pela linha que passa pelo marcador do trago ao marcador do maléolo externo e linha paralela ao fio de prumo que passa pelo marcador do maléolo externo) (Figura 1).

\section{Análise Estatística}

Os participantes foram caracterizados por meio da estatística descritiva e para cada medição foi considerada a média aritmética dos três avaliadores. Somente valores absolutos foram considerados. Foi utilizada a média e o 



Figura 1. Ângulos medidos no plano coronal: a) assimetria da cabeça, b) assimetria de ombros, c) assimetria pélvica, d) assimetria do trocanter, e) assimetria dos joelhos, $\mathrm{f}$ ) assimetria maleolar, e g) assimetria corporal. No plano sagital: h) ângulo sagital cervical, i) ângulo sagital dos ombros, e j) ângulo sagital corporal.

desvio padrão para as variáveis angulares calculadas sobre cada postura com pesos diferentes de mochila.

A normalidade e a esfericidade dos dados foram avaliadas, respectivamente, por meio dos testes de Kolmogorov-Smirnov e de Mauchly. Desta forma, para comparar os ângulos obtidos em cada postura com pesos diferentes de mochila foi utilizada Análise de Variância para Medidas Repetidas (variáveis que verificam a normalidade) e o teste não-paramétrico de Friedman (variáveis que não verificam a condição de normalidade). Mesmo com uma amostra igual a 50, quando os pressupostos de aplicabilidade da análise de variância paramétrica não são cumpridos, é mais adequado utilizar a análise não paramétrica. Havendo significância estatística foram realizadas múltiplas comparações aos pares para detectar onde estava a diferença.

A confiabilidade inter-avaliador foi analisada por meio do Coeficiente de Correlação Intraclasse (ICC).
Foi utilizado para análise o programa estatístico Statistical Package for the Social Sciences (SPSS) versão 17.0 para Windows e, para todos os procedimentos, foi adotado o nível de significância de $5 \%(p<.05)$ com distribuição bi-caudal.

\section{RESULTADOS}

O peso da mochila habitualmente utilizado pelos escolares foi $2.49 \pm 1.18 \mathrm{~kg}$ representando $6.79 \pm 3.37 \%$ do PC (41 mochilas apresentaram menos do que $10 \%$ do PC). A Tabela 2 mostra as medidas de tendência central e dispersão para os ângulos avaliados no plano coronal e sagital. A Análise de Variância para Medidas Repetidas mostrou diferenças estatisticamente significativas para as variáveis Ângulo Sagital Cervical $(p<.00)$ e Ângulo Sagital Corporal $(p<.00)$. O teste não-paramétrico de Friedman mostrou diferença somente para a variável Ângulo Sagital dos Ombros ( $p$ $<.01)$. As Comparações aos pares mostraram que na variável Ângulo Sagital Cervical houve diferença significativa entre SP vs $\mathrm{PH}(p=.01)$, SP vs $10 \%$ PC $(p=.001)$, SP vs $15 \%$ PC $(p=$ $.000)$, SP vs $20 \%$ PC ( $p=.000)$, PH vs $15 \%$ PC $(p=.01)$, PH vs $20 \%$ PC $(p=.000), 10 \%$ PC vs $20 \%$ PC $(p=.000)$ e $15 \%$ PC vs $20 \%$ PC ( $p=$ $.01)$; na variável Ângulo Sagital Corporal entre SP vs PH $(p=.000)$, SP vs $10 \%$ PC $(p=.000)$, SP vs $15 \%$ PC $(p=.000)$, SP vs $20 \%$ PC ( $p=$ $.000)$, PH vs $10 \%$ PC $(p=.03)$, PH vs $15 \%$ PC $(p=.000)$, PH vs $20 \%$ PC $(p=.000), 10 \%$ PC vs $15 \%$ PC ( $p=.000), 10 \%$ PC vs $20 \%$ PC ( $p$ $=.000)$ e $15 \%$ PC vs $20 \%$ PC $(p=.000)$; na variável Ângulo Sagital dos Ombros entre SP vs $15 \%$ PC $(p=.008)$, SP vs $20 \%$ PC $(p=.04)$, $\mathrm{PH}$ vs $15 \% \mathrm{PC}(p=.003)$, PH vs $20 \% \mathrm{PC}(p=$ $.03)$.

Foi encontrada alta confiabilidade inter-avaliador (ICC entre 0.83 a 0.99) para todas as medidas angulares avaliadas durante a postura SP de mochila, com PH e com $10 \%, 15 \%$ e $20 \%$ do PC $(p<.05)$ (Tabela 3). Os valores angulares medidos pelos três avaliadores apresen- 
taram os mesmos critérios e se mostraram consistentes. Os avaliadores se submeteram a um treino prévio e respeitaram um ambiente propício para a aquisição das imagens com privacidade, conforto, boa iluminação e temperatura controlada.

\section{DISCUSSÃO}

A mochila é a forma mais utilizada no transporte do material escolar e é essencial que haja cuidados tanto com a carga, como com a maneira de como esta é transportada pela criança (Goodgold et al., 2002; Rodrigues,

Tabela 2

Média e desvio-padrão dos ângulos (graus) observados durante a postura Sem Peso (SP) de mochila, c/Peso Habitualmente utilizado (PH) e com 10\%, 15\% e 20\% do Peso Corporal (PC) colocada nos dois ombros $(n=50)$

\begin{tabular}{|c|c|c|c|c|c|c|c|}
\hline & SP & $\mathrm{PH}(6.8 \% \mathrm{PC})$ & $10 \%$ PC & $15 \%$ PC & $20 \%$ PC & F ou $\chi^{2}$ & $p$ \\
\hline \multicolumn{8}{|l|}{ Plano Coronal } \\
\hline Assimetria Cabeça ${ }^{b}$ & $2.34(1.89)$ & $2.46(2.32)$ & $2.73(2.56)$ & $2.75(2.71)$ & $2.73(2.59)$ & 7.28 & .12 \\
\hline Assimetria Ombro ${ }^{b}$ & $1.96(1.51)$ & $1.99(1.52)$ & $2.26(2.08)$ & $2.36(1.67)$ & $2.44(1.74)$ & 3.73 & .44 \\
\hline Assimetria Pélvica b & $2.22(2.02)$ & $2.17(1.75)$ & $2.29(1.88)$ & $2.44(1.95)$ & $2.28(2.14)$ & 1.42 & .84 \\
\hline AssimetriaTrocanter ${ }^{b}$ & $3.12(2.99)$ & $2.98(2.31)$ & $3.66(2.52)$ & $3.37(2.67)$ & $3.47(3.04)$ & 6.63 & .16 \\
\hline Assimetria Joelho ${ }^{\text {a }}$ & $2.54(1.57)$ & $2.48(1.86)$ & $2.58(1.73)$ & $2.42(1.70)$ & $2.47(1.63)$ & 0.29 & .88 \\
\hline Assimetria Maleolar ${ }^{b}$ & $2.52(1.92)$ & $2.66(2.02)$ & $2.76(2.07)$ & $2.79(2.29)$ & $2.92(2.22)$ & 7.31 & .12 \\
\hline Assimetria Corporal b & $0.69(0.55)$ & $0.86(0.76)$ & $0.83(0.61)$ & $1.01(0.99)$ & $1.02(0.99)$ & 8.33 & .08 \\
\hline \multicolumn{8}{|l|}{ Plano Sagital } \\
\hline Ângulo S. Cervical a & $51.21(5.42)$ & $49.24(6.47)$ & $48.74(5.31)$ & $47.99(5.62)$ & $46.46(6.21)$ & 15.67 & $.00 *$ \\
\hline Ângulo S. Ombro b & 38) & $\begin{array}{l}133.77 \\
(16.04)\end{array}$ & 12207 & 12015 & 12008 & 14.54 & $.00^{*}$ \\
\hline Ângulo S. Corporal a & $4.13(1.15)$ & $5.41(1.45)$ & $5.81(1.25)$ & $6.51(1.44)$ & $7.30(1.94)$ & 63.44 & $.00^{*}$ \\
\hline
\end{tabular}



*Diferença estatisticamente significativa: Ângulo Sagital Cervical (SP vs PH, SP vs 10\% PC, SP vs $15 \%$ PC, SP vs $20 \%$ PC, PH vs $15 \%$ PC, PH vs $20 \%$ PC, $10 \%$ PC vs $20 \%$ PC, $15 \%$ PC vs $20 \%$ PC); Ângulo Sagital dos Ombro (SP vs $15 \%$ PC, SP vs $20 \%$ PC, PH vs $15 \%$ PC, PH vs $20 \%$ PC); Ângulo Sagital Corporal (SP vs PH, SP vs $10 \%$ PC, SP vs $15 \%$ PC, SP vs $20 \%$ PC, PH vs $10 \%$ PC, PH vs $15 \%$ PC, PH vs $20 \%$ PC, $10 \%$ PC vs $15 \%$ PC, $10 \%$ PC vs $20 \%$ PC, $15 \%$ PC vs $20 \%$ PC).

Tabela 3

Confiabilidade entre três avaliadores nas medidas angulares avaliadas durante a postura Sem Peso (SP) de mochila, c/Peso Habitualmente utilizado (PH) e com 10\%, 15\% e 20\% do Peso Corporal (PC) analisadas por meio do Coeficiente de Correlação Intraclasse (ICC) $(n=50)$

\begin{tabular}{ccccccc}
\hline & \multicolumn{7}{c}{ ICC } & & \\
\cline { 2 - 5 } & SP & PH & $10 \%$ PC & $15 \%$ PC & $20 \%$ PC & \\
\hline Plano Coronal & & & & & & \\
Assimetria Cabeça & 0.99 & 0.99 & 0.99 & 0.99 & 0.99 & $.00^{*}$ \\
Assimetria Ombro & 0.99 & 0.99 & 0.99 & 0.99 & 0.99 & $.00^{*}$ \\
Assimetria Pélvica & 0.99 & 0.99 & 0.99 & 0.99 & 0.99 & $.00^{*}$ \\
Assimetria Trocanter & 0.99 & 0.99 & 0.99 & 0.99 & 0.99 & $.00^{*}$ \\
Assimetria Joelho & 0.99 & 0.99 & 0.99 & 0.99 & 0.99 & $.00^{*}$ \\
Assimetria Maléolo & 0.99 & 0.99 & 0.96 & 0.99 & 0.99 & $.00^{*}$ \\
Assimetria Corporal & 0.96 & 0.90 & 0.97 & 0.96 & 0.97 & $.00^{*}$ \\
Plano Sagital & & & & & & \\
Ângulo Sagital Cervical & 0.99 & 0.99 & 0.98 & 0.99 & 0.99 & $.00^{*}$ \\
Ângulo Sagital Ombro & 0.99 & 0.99 & 0.98 & 0.99 & 0.95 & $.00^{*}$ \\
Ângulo Sagital Corporal & 0.83 & 0.95 & 0.93 & 0.95 & 0.98 & $.00^{*}$ \\
\hline
\end{tabular}

* estatisticamente significativo: $p<.01$ 
Montebelo, \& Teodori, 2008; Whittifielf et al., 2005). Neste estudo $18 \%$ das crianças avaliadas transportavam em suas mochilas cargas superiores a $10 \%$ do PC. Outros estudos encontraram 13.2\% (Wittifield et al., 2005), 44.09\% (Souza \& Santos, 2010) e 55\% (Goodgold et al., 2002) transportando sobrepeso. Quando a carga excede a capacidade de sustentação da musculatura, ocorre sobrecarga na coluna vertebral, podendo levar a alterações posturais, dor ou disfunção da coluna (Rebelatto, Caldas, \& De Vitta, 1991; Rodrigues et al., 2008).

Também deve ser considerada a capacidade física da criança, a forma, o tempo e a frequência de transporte da mochila (Al-Khabbaz et al., 2008; Mackie, Stevenson, Reid, \& Legg, 2005). A mochila no dorso ocasiona maior atividade do músculo reto abdominal, enquanto os músculos eretores da coluna diminuem a sua atividade (Motmans, Tomlow, \& Vissers, 2006). Estes autores constataram que distribuindo o peso igualmente na frente e atrás requer menos esforço muscular e sugerem o uso de uma mochila escolar dupla. A altura do posicionamento da mochila também deve ser considerada. A posição do centro da mochila à altura da cintura minimiza o deslocamento postural (Grimmer, Dansie, Milanese, Pirunsan, \& Trott, 2002). No presente estudo não foi controlada a altura da posição da mochila. Todos os escolares foram avaliados com a sua mochila, ou seja, na posição utilizada habitualmente.

Sabe-se que no plano coronal são esperados resultados angulares iguais a zero para caracterizar a simetria (Iunes et al., 2005). Quando os escolares posaram sem mochila, as variáveis que mais se aproximaram do valor zero, ou seja, estiveram mais alinhadas foram assimetria de cabeça, de ombro, de pelve e assimetria corporal. Pesquisas citam desnivelamento de ombros, inclinação cervical e desalinhamento pélvico como desvios posturais presentes nos escolares (Pinho \& Duarte, 1995; Penha, João, Casarotto, Amino, \& Penteado, 2005; Detsch et al., 2007; Santos et al., 2009). Estes estudos, no entanto, não dispõem de valores quantitativos a serem comparados com os encontrados neste estudo.

No plano coronal, apesar de se observar uma maior média de assimetria com o aumento do peso sustentado pelo escolar, na maioria das variáveis analisadas esta diferença não foi significativa. Chansirinukor, Wilson, Grimmer, e Dansie (2001) também encontraram maior assimetria do posicionamento da cabeça com o aumento do peso da mochila com diferença significativa ao comparar o uso de mochila sem carga $(0.8 \pm 2.9)$ e o uso da mesma com carga igual a $15 \%$ do PC $(2.1 \pm 2.9)$. Na análise cinemática da marcha de estudantes universitários foi encontrada maior assimetria pélvica durante transporte de 15\% do PC (Smith et al., 2006). Apesar da relevância do tema, poucos trabalhos estabelecem valores angulares no plano coronal relacionando assimetria e incremento de carga.

No plano sagital observou-se que o aumento do peso da mochila ocasionou diminuição do ângulo sagital cervical e do ângulo sagital do ombro e aumento do ângulo sagital corporal. Observou-se que quanto maior foi a carga maior foi a alteração postural. Este ajustamento postural é necessário para a manutenção da projeção horizontal do centro de gravidade do escolar dentro da base de suporte.

A diminuição do ângulo sagital cervical, indicando anteriorização da cabeça, foi observada em todos os incrementos de carga avaliados. A anteriorização da cabeça também foi observada em outros estudos durante o transporte de carga superior a $10 \%$ do PC (Souza \& Santos, 2010) e de $15 \%$ do PC (Chansirinukor et al., 2001). Contudo, Devroey, Jonkers, De Becker, Lenaerts e Spaepen (2007) não encontraram alterações angulares na postura da cabeça com o aumento de $5 \%, 10 \%$ e $15 \%$ do PC. Estes autores observaram outras alterações posturais como aumento da flexão do tórax, da anteriorização pélvica e da flexão do quadril, mas apenas com o peso da mochila a $10 \%$ e $15 \%$ do PC (Devroey et al., 2007). 
O aumento do ângulo sagital do ombro ocorre quando este está mais anteriorizado em relação a C7 e protuso (Neiva, Kikwood, \& Godinho, 2009). Neste estudo observou-se a diminuição do ângulo sagital do ombro nas diferentes posturas com o aumento do peso da mochila. A ação mecânica do peso da mochila promoveu o deslocamento anterior do corpo e da cabeça e, consequentemente, aproximou as referências C7 e acrômio. Como o deslocamento anterior do acrômio foi menor do que o deslocamento anterior de C7 observou-se a diminuição do ângulo.

A postura com a mochila sem peso e com peso habitual foi significativamente diferente da postura com $15 \%$ PC e com $20 \%$ PC. Somente as mochilas com peso de $15 \%$ e de $20 \%$ do PC determinaram modificações na postura dos ombros. Estes resultados não confirmaram os obtidos por Chansirinukor et al. (2001) que não encontraram efeito significativo na postura do ombro com o aumento de carga de $15 \%$ do PC. A diminuição do ângulo sagital do ombro também pode representar protrusão de ombro se a postura da cabeça está anteriorizada (Chansirinukor et al., 2001).

O aumento do ângulo sagital do corpo, indicando anteriorização corporal, foi observado em todas as porcentagens de peso de mochila relativas ao peso corporal. $\mathrm{O}$ aumento linear da anteriorização corporal corrobora com os resultados de outro estudo com peso da mochila de $3 \%, 5 \%$ e $10 \%$ do PC (Grimmer et al., 2002). Outros estudos consideram somente pesos superiores a $10 \%$ do PC como prejudiciais ao alinhamento postural, recomendaram que $\mathrm{O}$ peso da mochila não ultrapasse $17 \%$ do PC (Pascoe, Pascoe, Wang, Shum, \& Kim, 1997) ou $15 \%$ do PC, embora tenham sido observadas alterações posturais também com 10\% (Shasmin, Abu Osman, Razali, Usman, \& Wan Abas, 2007). Contudo, estas alterações posturais podem não ser dependentes de um percentual de peso de mochila maior do que $10 \%$ do PC. O aumento gradativo da anteriorização do corpo com o aumento do peso da mochila pode ocasionar alterações posturais.

As alterações biomecânicas como mudanças na postura do tronco e da atividade muscular podem ocasionar sintomas osteomusculares na área do tronco (Al-Khabbaz et al., 2008). As sinergias posturais compensatórias ocasionam alterações no alinhamento postural. Então, deve-se repensar sobre a validade do uso da mochila já que, mesmo com o uso de cargas inferiores a $10 \%$ do PC (6.79\% do PC), houve alterações significativas na postura da cabeça e no alinhamento postural. A identificação precoce e a prevenção dos fatores causais das alterações posturais podem contribuir para que estes sejam revertidos. Acredita-se que a má postura esteja relacionada com os maus hábitos nas atividades de vida diária e a postura adequada com hábitos posturais corretos na vida adulta.

Neste estudo, a alta confiabilidade inter-avaliador obtida por meio da fotogrametria, mostra ser este um método fidedigno para análise das assimetrias e desvios posturais. Corroborando este resultado, na análise postural de Iunes et al. (2005), observou-se confiabilidade aceitável (ICCs entre 0.71 e 0.79) para a maioria das medidas angulares avaliadas. Na avaliação postural de 29 variáveis posturais com utilização do software SAPO, somente quatro foram consideradas não aceitáveis (ICCs < .70) (Ferreira et al., 2010). Além disso, outros estudos também demonstraram a alta confiabilidade de técnicas fotogramétricas para a avaliação de amplitudes de movimento (Sato, Vieira, \& Gil Coury, 2003).

\section{CONCLUSÕES}

Diante das evidências encontradas no presente estudo, conclui-se que mochilas escolares com peso de $6.79 \%, 10 \%, 15 \%$ e $20 \%$ do PC ocasionam um aumento linear da anteriorização da cabeça e do corpo. Este estudo não sustenta a recomendação de que o peso da mochila escolar não deve exceder $10 \%$ do PC 
já que cargas inferiores também ocasionaram alterações posturais. Considera-se, ainda, a utilização da fotogrametria como um recurso de análise postural quantitativa confiável.

\section{Agradecimentos:}

Nada declarado.

\section{Conflito de Interesses:}

Nada declarado.

\section{Financiamento:}

Nada declarado.

\section{REFERÊNCIAS}

Al-Khabbaz, Y. S., Shimada, T., \& Hasegawa, M. (2008). The effect of backpack heaviness on trunk-lower extremity muscle activities and trunk posture. Gait Posture, 28(2), 297-302.

Chansirinukor, W., Wilson, D., Grimmer, K., \& Dansie, B. (2001). Effects of backpacks on students: Measurement of cervical and shoulder posture. Australian Journal of Physiotherapy, 47, 110-116.

Detsch, C., Luz, A. M. H., Candotti, C. T., Scotto de Oliveira, D., Lazaron, F., Guimarães, L. K., \& Schimanoski, P. (2007). Prevalência de alterações posturais em escolares do ensino médio em uma cidade no Sul do Brasil. Revista Panamericana de Salud Publica, 21 (4): 231-8.

Devroey, C., Jonkers, I., De Becker, A., Lenaerts, G., \& Spaepen, A. (2007). Evaluation of the effect of backpack load and position during standing and walking using biomechanical, physiological and subjective measures. Ergonomics, 50(5), 728-742.

Ferreira, E. A. G., Duarte, M., Maldonado, E. P., Burke, T. N., \& Marques, A. P. (2010). Postural assessment software (PAS/SAPO): validation and reliability. Clinics, 65(7), 675-681.

Goodgold, S., Corcoran, M., Gamache, D., Gillis, J., Guerin, J., \& Coyle, J. Q. (2002). Backpack use in children. Pediatric Physical Therapy, 14, 122-131.
Grimmer, K., Dansie, B., Milanese, S., Pirunsan, U., \& Trott, P. (2002). Adolescent standing postural response to backpack loads: a randomised controlled experimental study. BMC Musculoskeletal Disorders, 3(10), 1-10.

Hong, Y., \& Brueggmann, G. P. (2000). Changes in gait patterns in 10-year-old boys with increasing loads when walking on a treadmill. Gait Posture, 11(3), 254-259.

Iunes, D. H., Castro, F. A., Salgado, H. S., Moura, I. C., Oliveira, A. S, \& Bevilaqua-Grossi, D. (2005). Confiabilidade intra e interexaminadores e repetibilidade da avaliação postural pela fotogrametria. Revista Brasileira de Fisioterapia, $9(3), 327-34$.

Mackie, H. W., Stevenson, J. M., Reid, S. A., \& Legg, S. J. (2005). The effect of simulated school load carriage configurations on shoulder strap tension forces and shoulder interface pressure. Applied Ergonomics, 36, 199-206.

Moore, M. J., White, G. L., \& Moore, L. D. (2007). Association of relative backpack weight with reported pain, pain sites, medical utilization, and lost school time in children and adolescents. Journal of School Health, 77(5), 232-239.

Motmans, R. R. E .E., Tomlow, S., \& Vissers, D. (2006). Trunk muscle activity in different modes of carrying schoolbags. Ergonomics, 49(2), 127-138.

Neiva, P., Kikwood, R. N., \& Godinho, R. (2009). Orientation and position of head posture, scapula and thoracic spine in mouth-breathing children. International Journal of Pediatric Otorhinolaryngology, 73, 227-236.

Pascoe, D., Pascoe, E., Wang, T., Shum, M., \& Kim, K. (1997). Influence of carrying book bags on gaitcycle and posture of youths. Ergonomics, 40, 631-641.

Penha, P. J., João, S. M. A., Casarotto, R. A., Amino, C. J., \& Penteado, D. C. (2005). Postural assessment of girls between 7 and 10 years of age. Clinics, 60(1), 9-16.

Pinho, R.A., \& Duarte, M.F.S. (1995). Análise postural em escolares de Florianópolis - SC. Revista Brasileira de Atividade Física e Saúde, 1(2): 
49-58.

Rebelatto, J. R., Caldas, M. A. J., \& De Vitta, A. (1991). Influência do transporte do material escolar sobre a ocorrência de desvios posturais em estudantes. Revista Brasileira de Ortopedia, 26(11/12), 403-410.

Rodrigues, S., Montebelo, M. I. L., \& Teodori, R. M. (2008). Distribuição da força plantar e oscilação do centro de pressão em relação ao peso e posicionamento do material escolar. Revista Brasileira de Fisioterapia, 12(1), 43-48.

Santos, C. I. S., Cunha, A. B. N., Braga, V. P., Saad, I. A. B., Ribeiro, M. Â. G. O., Conti, P. B. M., \& Oberg, T. D. (2009). Ocorrência de desvios posturais em escolares do ensino público fundamental de Jaguariúna, São Paulo. Revista Paulista de Pediatria, 27(1), 74-80.

Sato, T. O., Vieira, E. R., \& Gil Coury, H. (2003). Análise da confiabilidade de técnicas fotogramétricas para medir a flexão anterior do tronco. Revista Brasileira Fisioterapia, 7(1), 53-99.

Seven, Y. B., Akalan, N. E., \& Yucesoy, C. A. (2008). Effects of Back Loading on the Biomechanics of Sit-to-stand Motion in Healthy Children. Human Movement Science, 27(1), 65-79.
Shasmin, H. N., Abu Osman, N. A., Razali, R., Usman, J., \& Wan Abas, W. A. B. (2007). The Effect of Load Carriage Among Primary School Boys: A Preliminary Study. Journal of Mechanics in Medicine and Biology, 7(3), 265-274.

Smith, B., Ashton, K. M., Bohl, D., Clark, R. C., Metheny, J. B., \& Klassen, S. (2006) Influence of carrying a backpack on pelvic tilt, rotation, and obliquity in female college students. Gait Posture, 23, 263-267.

Souza, A. C. S., \& Santos, G. M. A. (2010). Influência do Peso da Mochila nas Alterações Posturais em Pré-Adolescentes. Terapia Manual, 8(38), $277-284$

Whittifielf, J., Legg, S. J., \& Hedderley, D. I. (2005). Schoolbag Weight and Musculoskeletal Symptoms in New Zealand Secondary Schools. Applied Ergonomics, 36, 193-198.

(cc) EY-No Todo o conteúdo da revista Motricidade está licenciado sob a Creative Commons, exceto quando especificado em contrário e nos conteúdos retirados de outras fontes bibliográficas. 